\title{
Adolescent and adult rats differ in the amnesic effects of acute ethanol in two hippocampus-dependent tasks: Trace and contextual fear conditioning
}

\author{
Pamela S. Hunt \\ Coll William \& Mary, Dept Psychol, Williamsburg, VA 23187 USA \\ Robert C. Barnet \\ Coll William \& Mary, Dept Psychol, Williamsburg, VA 23187 USA
}

Follow this and additional works at: https://scholarworks.wm.edu/aspubs

\section{Recommended Citation}

Hunt, P. S., \& Barnet, R. C. (2016). Adolescent and adult rats differ in the amnesic effects of acute ethanol in two hippocampus-dependent tasks: Trace and contextual fear conditioning. Behavioural brain research, $298,78-87$.

This Article is brought to you for free and open access by the Arts and Sciences at W\&M ScholarWorks. It has been accepted for inclusion in Arts \& Sciences Articles by an authorized administrator of W\&M ScholarWorks. For more information, please contact scholarworks@wm.edu. 
Research report

\title{
Adolescent and adult rats differ in the amnesic effects of acute ethanol in two hippocampus-dependent tasks: Trace and contextual fear conditioning
}

\author{
Pamela S. Hunt*, Robert C. Barnet \\ Department of Psychology, College of William \& Mary, United States
}

\section{H I G H L I G H T S}

- Acute ethanol interferes with hippocampal function.

- Adolescents and adults differ in sensitivity to ethanol.

- Adolescents show ethanol disruption of trace conditioning.

- Adults show ethanol disruption of context conditioning.

- Age differences in cognitive impairments by acute ethanol.

\section{A R T I C L E I N F O}

\section{Article history:}

\section{Received 5 April 2015}

Received in revised form 22 June 2015

Accepted 23 June 2015

Available online 17 July 2015

\section{Keywords:}

Adolescence

Alcohol

Trace conditioning

Context conditioning

Fear conditioning

Freezing

Hippocampus

\begin{abstract}
A B S T R A C T
Experience-produced deficits in trace conditioning and context conditioning have been useful tools for examining the role of the hippocampus in learning. It has also been suggested that learning in these tasks is especially vulnerable to neurotoxic effects of alcohol during key developmental periods such as adolescence. In five experiments we systematically examined the presence and source of age-dependent vulnerability to the memory-disrupting effects of acute ethanol in trace conditioning and contextual fear conditioning. In Experiment 1a pre-training ethanol disrupted trace conditioning more strongly in adolescent (postnatal day, PD30-35) than adult rats (PD65-75). In Experiment 1b when pre-training ethanol was accompanied by pre-test ethanol no deficit in trace conditioning was observed in adolescents, suggesting that state-dependent retrieval failure mediated ethanol's disruption of trace conditioning at this age. Experiment $2 \mathrm{a}$ and $\mathrm{b}$ examined the effect of ethanol pretreatment on context conditioning. Here, adult but not adolescent rats were impaired in conditioned freezing to context cues. Experiment $2 \mathrm{c}$ explored state-dependency of this effect. Pre-training ethanol continued to disrupt context conditioning in adults even when ethanol was also administered prior to test. Collectively these findings reveal clear age-dependent and task-dependent vulnerabilities in ethanol's disruptive effects on hippocampusdependent memory. Adolescents were more disrupted by ethanol in trace conditioning than adults, and adults were more disrupted by ethanol in context conditioning than adolescents. We suggest that adolescents may be more susceptible to changes in internal state (state-dependent retrieval failure) than adults and that ethanol disrupted performance in trace and context conditioning through different mechanisms. Relevance of these findings to theories of hippocampus function is discussed.
\end{abstract}

(ㄷ) 2015 Elsevier B.V. All rights reserved.

\section{Introduction}

Ethanol, depending upon dose, can have disruptive effects on learning and memory [1]. Interestingly, ethanol's effects depend

* Corresponding author at: Department of Psychology, College of William \& Mary, PO Box 8795, Williamsburg, VA 23187-8795 USA. Fax: +1 7572213896.

E-mail address: pshunt@wm.edu (P.S. Hunt). on the type of memory assessed. In humans, implicit memory may be impervious to acute ethanol and is generally immune to ethanol-induced amnesia [2]. In contrast, acute ethanol impairs the acquisition of explicit, declarative memory and ethanol-induced deficits in declarative recall are often reported [2,3]. In non-human subjects, acute ethanol can also interfere with learning and memory in a task-dependent manner. Available reviews of this extensive literature suggest that ethanol has markedly detrimental effects on hippocampus-dependent forms of learning and memory [4,5]. 
For example, in adult rats ethanol dose-dependently compromises performance on spatial memory tasks, such as the Morris water maze [6,7]. Weitemier and Ryabinin [8] showed that ethanol disrupted both trace and contextual fear conditioning in adult mice, while having no effect on delay conditioning (see also [9]). Similar effects of ethanol on contextual fear learning in rats have also been reported $[10,11]$. Importantly, these tasks that are affected by acute ethanol (spatial memory, trace conditioning, context conditioning) are known to involve the hippocampus. Performance on non-hippocampus variations of these tasks (delay conditioning, nonspatial/cue learning) is generally not affected by low to moderate doses of ethanol, although high doses can produce more general disruptions to learning $[8,12,13]$.

While the literature presented above indicates that acute ethanol can produce substantial deficits in some types of memory in adults, less is known about age-related differences in sensitivity to ethanol-induced learning impairments [14,15]. Available evidence is mixed. Some research suggests that adolescents are more sensitive than adults to the amnesic effects of ethanol, while others report that adolescents are lesssensitive. For example, Markwiese et al. [16] found that adolescent rats were more impaired in acquisition of the Morris water maze spatial task by a moderate $(1$ or $2 \mathrm{~g} / \mathrm{kg}$ ) dose of ethanol than were adults. Chin et al. [17] showed that adolescents and adults were equally impaired in the water maze task when ethanol was acutely administered prior to a test for spatial memory. Land and Spear [18] reported a greater disruptive effect of ethanol in adolescents, compared to adults, on an appetitive odor discrimination task. These particular age-dependent differences in ethanol effects on memory may relate to developmental changes in ethanol's disruption of hippocampal activity. Ethanol is known to suppress the firing rate of pyramidal neurons, disrupt hippocampal theta rhythm, antagonize NMDA receptor sub-types and decrease glutamate release (see [15]). Moreover, Swartzwelder and colleagues [19,20,21] reported that hippocampal slices from juvenile and adolescent rats showed greater sensitivity to ethanol inhibition of both NMDA-mediated synaptic plasticity and induction of long-term potentiation than slices obtained from adults.

Not all of the data however, support the idea that adolescents are more sensitive to the amnesic effects of acute ethanol. Land and Spear [13] reported that adult rats exhibited greater disruption in fear conditioning than adolescents. This was evident in reduced conditioned freezing to both a tone conditioned stimulus (CS) and contextual cues. The finding that cue (CS) conditioned responding was impaired by a moderate dose of ethanol $(1 \mathrm{~g} / \mathrm{kg})$ is perplexing, given that several other studies find no effect of ethanol on this type of fear conditioning with comparable doses [8,11]. In addition, Broadwater and Spear [22] recently reported a greater disruptive ethanol effect in adult rats, compared with adolescents, in contextual fear conditioning. Unlike the Land and Spear [13] results, no effect of ethanol was observed on CS conditioning at either age, a finding consistent with the reports cited above.

The primary question addressed in the present experiments was whether adolescents and adults are differentially sensitive to the memory-impairing effects of acute ethanol using two tasks known to require hippocampal function (trace and contextual fear conditioning). The literature concerning the effects of acute administration of alcohol on trace conditioning, one widely recognized form of hippocampus-dependent memory, is surprisingly limited. Only two studies have, to our knowledge, addressed this question. McKinzie et al. [10] used preweanling (17-day-old) rats and a study by Weitemier and Ryabinin [8] employed adult mice. Both reported detrimental effects of acute ethanol on trace, but not delay, fear conditioning. Another study by Melia et al. [11] examined the effects of acute alcohol on contextual fear conditioning, and alcohol was found to dose-dependently impair contextual fear conditioning in adult rats. The present experiments specifically compared the sensitivity of adolescent and adult rats to the effects of acute pre-training ethanol on delay, trace, and contextual fear conditioning in the same series of studies. Given that adolescent rats are more impaired by alcohol on hippocampusdependent spatial learning tasks than are adults [16] and that adults may be more sensitive to ethanol in contextual fear conditioning [22], we expected that age-related differences in sensitivity to ethanol amnesia would be obtained in both trace and contextual fear conditioning but given existing discrepancies in the literature the exact direction of age-related vulnerability was not initially hypothesized.

Pre-training drug administration can result in state-dependent performance deficits that do not reflect deficits in learning or memory acquisition per se [23]. Therefore, a second goal of the present research was to systematically explore the contribution of statedependent retrieval failure to any ethanol-induced deficits in trace and context conditioning, when observed (see [11]). Finally, none of the research previously exploring age differences in the effects of acute ethanol has permitted evaluation of sex differences. Most prior studies have employed only male subjects and the two that included both sexes $[13,18]$ did not analyze the data for possible sex differences. In the present work, we extend the available literature further by including both males and females in order to assess whether age-dependent vulnerabilities in ethanol's memory impairing effects also depend on sex.

\section{General method}

\subsection{Subjects}

A total of 409 Sprague-Dawley-derived rats served as subjects in these experiments. Two hundred and fifteen animals were trained and tested as adolescents (range 30-35 days, derived from 29 litters) and 194 were trained and tested as adults (range 65-75 days, derived from 38 litters). Approximately equal numbers of male and female subjects were included in each treatment group whenever possible (all ns $=8-10$ ). In each experiment, no more than one male and one female from a litter were assigned to each treatment group.

Subjects were born and raised at the College of William and Mary (Williamsburg, VA) in the Psychology Department's vivarium. Breeder animals (Charles River Laboratories, Wilmington, MA) were housed in $50.8 \times 40.6 \times 21.6 \mathrm{~cm}$ clear polycarbonate cages with pine chip bedding and wire tops. All animals had free access to water and high-protein rodent pellets (LabDiet Formula 5008). Cages were checked at $1000 \mathrm{~h}$ daily for pups, and the day of birth was designated Postnatal Day (PD) 0. On PD 2, litters were culled to 8-10 pups that remained with the dam until PD 21. At weaning, animals were housed with same-sex siblings in identical cages. On PD 40 animals were pair housed. The vivarium light:dark schedule was maintained at $14: 10 \mathrm{~h}$ with light onset at $0600 \mathrm{~h}$. All experimental procedures were carried out during the light portion of the cycle and were approved by the College of William and Mary's Institutional Animal Care and Use Committee that follows guidelines established by the NIH.

\subsection{Apparatus}

All delay and trace conditioning (Experiment $1 \mathrm{a}$ and $\mathrm{b}$ ), and context conditioning (Experiment 2a, b, and c), occurred in two identical $38.0 \times 26.0 \times 22.0 \mathrm{~cm}$ modified Skinner boxes. Two of the four walls were made of clear Plexiglas and the other two were made of aluminum. The floor was constructed of $5 \mathrm{~mm}$ stainless steel rods spaced $1.5 \mathrm{~cm}$ apart (center to center). In all experiments 
the US was a $0.5 \mathrm{~mA}, 1 \mathrm{~s}$ shock delivered through the grid floor by a custom built shock generator. In Experiment $1 \mathrm{a}$ and $\mathrm{b}$ the CS for trace and delay conditioning was produced by a $25-\mathrm{W}$ white bulb that flashed at a rate of $2 / \mathrm{s}$. The center of the bulb was located $12 \mathrm{~cm}$ above the floor and $8.5 \mathrm{~cm}$ from one of the two clear walls of the chamber. In Experiment $2 \mathrm{~b}$ and $\mathrm{c}$ the tone used for background context conditioning (discussed later) was an $80 \mathrm{~dB}, 1600 \mathrm{~Hz}$ pure tone that pulsed at a rate of $2 / \mathrm{s}$ (rise-fall time $250 \mathrm{msec}$ ). The 4-in speaker for the tone was mounted in the ceiling of the training chamber. The training chambers were individually housed in sound-attenuating shells measuring $67.0 \times 71.5 \times 71.0 \mathrm{~cm}$. On the inside of each shell was a 4 -W red bulb to provide constant lowlevel illumination. A PC computer was used to interface Coulbourn Instruments (Allentown, PA) software and hardware, which controlled all stimulus presentations.

All CS testing occurred in a novel context located in another room of the laboratory. During CS testing subjects were tested individually in one of two identical $29.0 \times 21.5 \times 46.5 \mathrm{~cm}$ clear Plexiglas chambers that were open at the top and bottom. The bottom $11 \mathrm{~cm}$ of each chamber was cut out and fitted with horizontally mounted stainless steel bars $0.5 \mathrm{~mm}$ in diameter and spaced $1.5 \mathrm{~cm}$ apart. The chambers were placed onto Plexiglas floors that were covered with brown paper. The chambers were housed in soundattenuating shells (IAC; Industrial Acoustics, NY) with a white, 7-W bulb mounted on one of the inner walls. The CS used during the test was identical to that of training. In trace conditioning Experiments $1 \mathrm{a}$ and $1 \mathrm{~b}$ the test CS was a flashing light and in context conditioning Experiment $2 \mathrm{~b}$ the test $\mathrm{CS}$ was the pulsing tone presented through a JBL speaker mounted in the ceiling of the sound-attenuating shell. All context testing in Experiment 2a, b, and c (freezing to the context) occurred in the same context as training. All test sessions were videotaped using Sony video cameras (Model CCD-TRV67).

\subsection{Procedure}

Subjects within each age were randomly assigned to treatment groups, with approximately equal males and females in each (ns $=8-10)$. Specific treatment conditions are described in each experiment.

\section{Experiment 1a: delay vs. trace fear conditioning}

There are many variants of the classical conditioning paradigm. In delay conditioning, the onset of the unconditioned stimulus (US) occurs at the termination of the conditioned stimulus (CS). The amygdala is the brain structure most often associated with delay fear conditioning [24]. In trace conditioning, the offset of the CS and onset of the US are separated by a stimulus-free period known as the trace interval. Interference with normal hippocampal function, via lesion or pharmacological treatment, has been shown to affect acquisition of trace conditioning, while sparing delay [25-29]. Squire and colleagues [30,31] argue that trace conditioning taps into a hippocampal declarative memory system, which supports the conjecture that trace conditioning can be used in animal research to explore declarative memory impairments. If functional changes in the hippocampus result from acute ethanol intoxication we would expect subjects to be deficient in acquisition of trace conditioning but not delay conditioning (cf. [8]). Experiment 1a compared the effects of acute ethanol on trace and delay conditioning in adolescent and adult animals.

\section{Method}

\subsection{Subjects E' apparatus}

One hundred and eighty Sprague-Dawley-derived rats were used ( 100 adolescents, $n s=10$ and 80 adults, $n s=8$ ). The apparatus as described in the General Method was used.

\subsection{Procedure}

Treatment groups were defined on the basis of conditioning treatment (trace, delay, or unpaired) and ethanol dose $(0,0.5,1.0$ or $1.5 \mathrm{~g} / \mathrm{kg}$ ). Doses of ethanol were achieved by varying the volume of a $20 \% \mathrm{v} / \mathrm{v}$ solution with tap water as the vehicle. Solutions were prepared fresh daily. Animals in the trace and delay conditioning groups were intragastrically (i.g.) administered one of the four ethanol doses prior to training. Unpaired subjects were administered either 0 or $1.5 \mathrm{~g} / \mathrm{kg}$ ethanol prior to training. Following ethanol administration animals were placed in pairs in holding cages lined with pine chips.

Thirty minutes after ethanol administration, subjects were placed into the conditioning chambers and given a 5-min adaptation period. This was followed by 5 CS and 5 US presentations. Animals in delay conditioning groups were given 5 pairings of a 20 -sec light CS that terminated with onset of a $0.5 \mathrm{~mA}, 1-\mathrm{s}$ footshock. For the trace groups, subjects were given 5 trials in which presentation of a 10 -s CS was followed by a 10 -s trace interval that terminated with shock. Thus both groups had equivalent interstimulus intervals, defined as the time from CS onset to US onset (see also [32]). Finally, for unpaired groups the 10-s light CSs and shock USs were explicitly unpaired, with the stipulation that a US could not occur $60 \mathrm{~s}$ prior to or following a CS. Inter-trial intervals (US-to-CS interval) ranged from 200 to $300 \mathrm{~s}$ for all groups and the training session lasted for $30 \mathrm{~min}$.

Testing occurred approximately $24 \mathrm{~h}$ after training. Animals were placed into the same holding cages as used on the training day, although no drug was given. After $30 \mathrm{~min}$ in the holding cage, subjects were tested for conditioning to the light CS in a novel context. After a 5-min acclimation period subjects were given three nonreinforced CS presentations, and CS duration was the same as that used in training. Test sessions were videotaped for later scoring of freezing. Freezing, defined as the absence of movement except that required for respiration [33] was scored by an observer blind to ethanol treatment using a time-sampling procedure. Freezing was scored for $10 \mathrm{~s}$ prior to each CS presentation (pre-CS freezing) and for a 20-s period beginning with CS onset (CS freezing). For delay groups, the CS was present during the entire CS period. For trace and unpaired groups the CS was present during the first $10 \mathrm{~s}$ of the CS period. Freezing after CS offset was scored because this corresponded to the trace interval present during training in the trace groups. Freezing was scored at $2 \mathrm{~s}$ intervals during each epoch. After initial analyses of pre-CS freezing, the data were converted into a Change score (\% CS freezing - \% pre-CS freezing) which reflected CS-elicited freezing.

\section{Results and discussion}

Adolescent rats were severely disrupted in acquisition of hippocampus-dependent trace fear conditioning at all pre-training ethanol doses while delay conditioning was unaffected. By contrast, pre-training ethanol failed to affect trace conditioning in adults at lower doses $(.5 \mathrm{~g} / \mathrm{kg}, 1.0 \mathrm{~g} / \mathrm{lg})$ and impaired trace conditioning only at the highest dose $(1.5 \mathrm{~g} / \mathrm{kg})$. No disruption of delay conditioning in adults was observed. These findings with rats are generally consistent with those of Weitemeier and Ryabinin [8], who reported that trace but not delay fear conditioning was disrupted in mice when animals were trained under the acute effects of ethanol.

\subsection{Pre-CS freezing}

\subsubsection{Unpaired groups}

Percent freezing recorded during the $10 \mathrm{~s}$ prior to CS onset for Unpaired groups was analyzed using a 2 (age) $\times 2$ (dose) $\times 2$ 
$($ sex $) \times 3$ (trial) mixed-factor ANOVA. The analysis yielded no significant main effects or interactions. Overall, pre-CS freezing was low (Mean $\pm \mathrm{SEM}=22.5 \% \pm 7.6$ ) and was unaffected by prior ethanol administration.

\subsubsection{Delay and trace groups}

Percent pre-CS freezing for Delay and Trace groups was analyzed using a 2 (age) $\times 2$ (conditioning) $\times 4$ (dose) $\times 2($ sex $) \times 3$ (trial) mixed-factor ANOVA. The analysis yielded significant main effects of age and trial, as well as Conditioning $\times$ Trial, and Age $\times$ Dose $\times$ Sex $\times$ Trial interactions [smallest $F(6,224)=2.82$, $p<.05]$. In general, pre-CS freezing increased during the test session, especially in the subjects trained with the Delay procedure. This increase was also especially pronounced in adult males. Because increases in pre-CS freezing can obscure the measure of CS-elicited freezing (Change scores), all subsequent analyses were conducted on the data obtained from the first test trial only. A 2 (age) $\times 2$ (conditioning) $\times 4$ (dose) $\times 2$ (sex) ANOVA conducted on the pre-CS freezing data for Trial 1 yielded only a main effect of age, $F(1,112)=5.29, p<.05$. Adolescent animals showed slightly more pre-CS freezing $(M=13.8 \pm 3.0 \%)$ than adults $(M=3.44 \pm 3.3 \%)$.

\subsection{CS freezing}

\subsubsection{Unpaired groups}

Responding to the CS on Trial 1 (Change scores) by unpaired controls was analyzed with a 2 (age) $\times 2$ (dose) $\times 2($ sex $)$ ANOVA. The analysis yielded no significant effects or interactions. Unpaired animals of each age showed virtually no CS-elicited freezing, and scores were unaffected by pre-training ethanol administration. In subsequent statistical analyses (see below), the unpaired groups given either 0 or $1.5 \mathrm{~g} / \mathrm{kg}$ ethanol were combined into a single control.

\subsubsection{Delay and trace groups}

Change scores obtained for Trial 1 were analyzed using a 2 (age) $\times 2$ (conditioning) $\times 4$ (dose) $\times 2$ (sex) between-groups ANOVA. Analyses revealed a main effect of conditioning [ $F$ $(1,112)=40.26, p<.001]$, an Age $\times$ Dose $\times$ Sex interaction $[F(3$, $112)=4.26, p<.01]$ and a Conditioning $\times$ Dose $\times$ Sex interaction $[F$ $(3,112)=2.80, p<.05]$. In order to explore these interactions further, follow-up ANOVAs were conducted separately for each age and conditioning group as presented below.

\subsubsection{Adolescents}

5.2.3.1. Delay conditioning. CS-elicited freezing to the Delay CS was robust and was unaffected by ethanol (see Fig. 1a). The data were analyzed using a 5 (group; 4 ethanol doses plus unpaired control) $\times 2$ (sex) ANOVA which yielded a main effect of group, $F(4$, $50)=21.31, p<.001$, but no effects of sex. All animals trained with the Delay procedure, regardless of ethanol dose, exhibited high levels of CS-elicited freezing, and all conditioned groups differed from the combined Unpaired control.

5.2.3.2. Trace conditioning. Trace conditioning was severely disrupted by pre-training ethanol in adolescent subjects (Fig. 1a). A 5 (group) $\times 2$ (sex) ANOVA yielded a main effect of group, $F$ $(4,50)=5.09, p<.01$. Post hoc comparisons (Newman-Keuls tests, $p<.05)$ indicated that the subjects given $0 \mathrm{~g} / \mathrm{kg}$ ethanol exhibited greater freezing to the Trace CS than the unpaired controls. Moreover, ethanol interfered with trace conditioning at all doses. Groups trained with $0.5,1.0$ or $1.5 \mathrm{~g} / \mathrm{kg}$ ethanol each showed significantly less CS-elicited freezing than the $0 \mathrm{~g} / \mathrm{kg}$ group, and none of the ethanol groups differed from unpaired controls.
5.2.4. Adults

5.2.4.1. Delay conditioning. Freezing observed to the Delay CS was unaffected by pre-training ethanol administration (see Fig. 1b). A 5 (group) $\times 2$ (sex) ANOVA revealed a main effect of group, $F$ (4, $38)=10.18, p<.001$. Post hoc Newman-Keuls tests supported the conclusion that all Delay groups differed from the unpaired control, but did not differ from each other.

5.2.4.2. Trace conditioning. A 5 (group) $\times 2$ (sex) ANOVA yielded a significant effect of group, $F(4,38)=4.40, p<.01$. Post hoc comparisons indicated that the adult subjects given $1.5 \mathrm{~g} / \mathrm{kg}$ ethanol prior to training exhibited less CS-elicited freezing than all other trace groups and did not differ from unpaired controls (Fig. 1b). Thus, the adult subjects were affected by ethanol, but only at the highest dose examined $(1.5 \mathrm{~g} / \mathrm{kg})$.

\section{Experiment 1b: state-dependent retention in adolescents}

The previous experiment indicated that adolescent subjects were more sensitive than adults to the acute effects of ethanol on trace conditioning. Adolescents showed a consistent deficit in trace conditioning regardless of ethanol dose. It was also apparent that adolescents showed impaired trace conditioning at lower doses than adults, consistent with the idea that adolescents are more vulnerable to the memory-impairing effects of ethanol than are adults. While these data suggest that ethanol impaired trace conditioning by disrupting memory encoding functions of the hippocampus, an alternative explanation is also viable. The change in internal state from training (ethanol present) to testing (ethanol absent) may have influenced memory retrieval or behavioral expression of the previously acquired memory. This possibility is known as state-dependent retrieval [23]. In order to evaluate the effects of a change in internal state on trace conditioned responding, Experiment $1 \mathrm{~b}$ manipulated the presence or absence of ethanol during both training and testing. Because the disruption in trace conditioning was more apparent in adolescent than adult subjects, only adolescents were tested here. Four groups of animals were trained and tested in the trace conditioning task described in Experiment 1a but were pretreated before conditioning with either water or ethanol, and, were also pretreated with water or ethanol prior to test. If ethanol pretreatment disrupts memory encoding, then both groups exposed to ethanol before conditioning should perform more poorly than groups exposed to water prior to conditioning. If the ethanol-mediated impairment in trace conditioning seen in adolescent animals from Experiment $1 \mathrm{a}$ is due to a state-dependent retrieval deficit, then trace conditioning should be impaired when animal are trained and tested with different pretreatments but not when trained and tested with the same pretreatment. That is, ethanol pretreatment should not impair trace conditioning provided that animals are tested in the same state.

\section{Method}

\subsection{Subjects $\mathcal{E}$ apparatus}

Thirty-six adolescent male and female rats were used. One animal assigned to group Water-Water described below was not tested because of an improper intubation prior to test, resulting in $n=8$ in group Water-Water and $n=9$ in all other groups. The apparatus as previously described was used.

\subsection{Procedure}

Animals were randomly assigned to one of four groups differentiated by whether they were given water or ethanol prior to training 

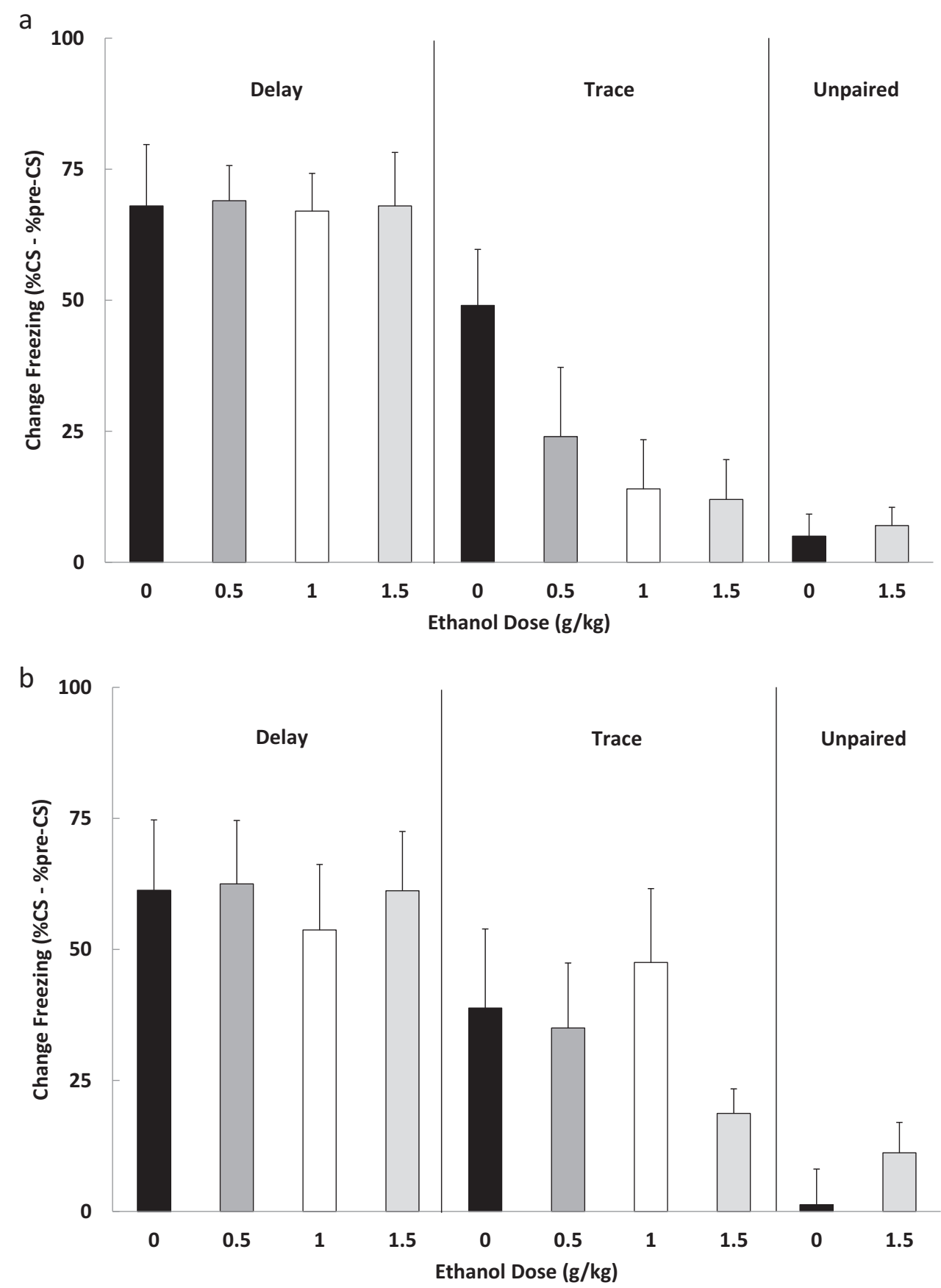

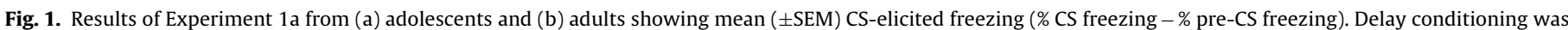

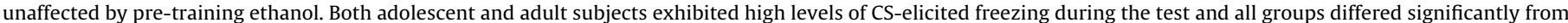

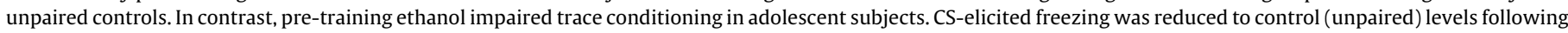

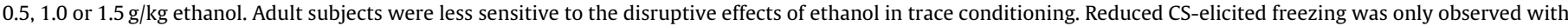
the highest $(1.5 \mathrm{~g} / \mathrm{kg}$ ) dose of ethanol in animals of this age.

and whether they were given water or ethanol prior to test. The ethanol dose was either $0 \mathrm{~g} / \mathrm{kg}$ (water) or $1.0 \mathrm{~g} / \mathrm{kg}$ and was administered i.g. $30 \mathrm{~min}$ prior to training and/or testing. The higher dose $(1.5 \mathrm{~g} / \mathrm{kg})$ was not used here because it was observed to produce sedation and hypoactivity that would interfere with our measure of CS-elicited freezing at test (see also [22]). We observed no general sedation in Experiment 1a following $1.0 \mathrm{~g} / \mathrm{kg}$ ethanol. Groups Water-Water and Water-Ethanol received water $(0 \mathrm{~g} / \mathrm{kg})$ pretreatment prior to the conditioning session and received either water (Water-Water) or ethanol (Water-Ethanol) pretreatment prior to the test. Groups Ethanol-Water and Ethanol-Ethanol received ethanol $(1.0 \mathrm{~g} / \mathrm{kg})$ pretreatment prior to conditioning and received either water (Ethanol-Water) or ethanol (Ethanol-Ethanol) pretreatment prior to test. All animals were trained and tested for trace conditioning as in Experiment 1a.

\section{Results and discussion}

Adolescent animals trained under acute ethanol intoxication performed poorly but only when tested in a drug-free state. When ethanol was administered both before conditioning and before test, no evidence of impaired trace conditioning was observed. 


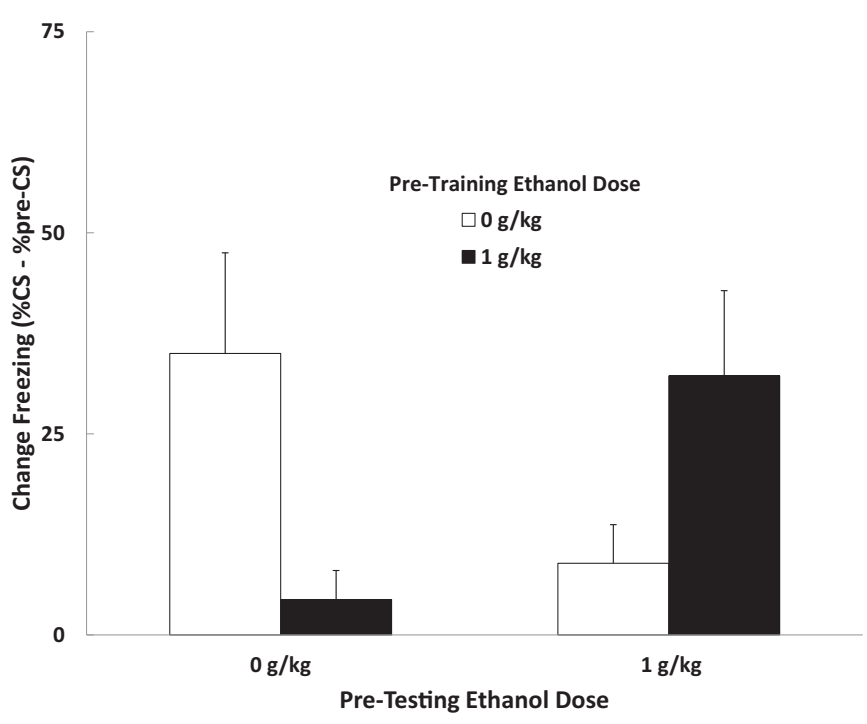

Fig. 2. Results from Experiment 1b showing the effects of pre-training and/or pretesting ethanol administration on trace conditioning in adolescent (PD 30-35) rats. Mean $( \pm$ SEM) CS-elicited freezing (\%CS freezing - \%pre-CS freezing). Adolescent animals show impaired trace conditioned responding when there was a change in drug state between training and testing but no impairment in trace conditioning when drug state was the same in training and test.

\subsection{Pre-CS freezing}

The 2 (training dose) $\times 2$ (testing dose) $\times 2$ (sex) ANOVA conducted on pre-CS freezing recorded prior to the first test trial revealed no significant effects. Animals showed low levels of freezing $(M=9.71 \pm 4.5 \%)$ in the novel test context prior to the first CS presentation.

\subsection{CS freezing}

Change scores obtained for the first test trial were analyzed using a 2 (training dose) $\times 2$ (testing dose) $\times 2$ (sex) ANOVA that revealed a significant Training Dose $\times$ Testing Dose interaction, $F$ $(1,31)=11.51, p<.01$. Neither main effect related to ethanol dose was significant and there were no effects of sex. The data are shown in Fig. 2. As in Experiment 1a, adolescent rats exposed to pre-training ethanol were impaired in trace conditioning. Group Ethanol-Water froze less to the trace CS than Group Water-Water. However, the ethanol-produced deficit in trace conditioned was reversed when animals were also exposed to pre-test ethanol. Under these conditions, no evidence of a trace conditioning deficit was observed. Group Ethanol-Ethanol expressed just as much conditioned freezing to the trace CS as Group Water-Water, despite ethanol pretreatment. This result implies that some of the disruption in trace conditioning produced by pre-training ethanol administration observed in Experiment $1 \mathrm{a}$ was the result of a shift in internal state between training and test (state-dependent retrieval failure) rather than an effect of ethanol on memory encoding per se.

\section{Experiment 2a: contextual fear conditioning}

Pre-training ethanol administration has been shown to disrupt contextual fear conditioning which is another widely recognized hippocampus-dependent task $[8,9,11,22]$. Animals trained following lesions of the hippocampus, or pharmacological blockade of muscarinic cholinergic receptors, fail to exhibit conditioned freezing to the context when tested after a $24 \mathrm{~h}$ or longer retention interval [34,35]. The purpose of Experiment 2a was to examine
Table 1

Mean $( \pm$ SEM) percent freezing to the context in adolescent and adult rats in Experiment 2 a. Subjects were administered $0,0.5,1.0$ or $1.5 \mathrm{~g} / \mathrm{kg}$ ethanol i.p. prior to context conditioning. Subjects were tested for context freezing $24 \mathrm{~h}$ later. No drug was given prior to test.

\begin{tabular}{lllll}
\hline \multicolumn{4}{l}{ Pre-training ethanol dose } \\
\hline Age & $0 \mathrm{~g} / \mathrm{kg}$ & $0.5 \mathrm{~g} / \mathrm{kg}$ & $1.0 \mathrm{~g} / \mathrm{kg}$ & $1.5 \mathrm{~g} / \mathrm{kg}$ \\
\hline 35 & 56 & 54 & 60 & 59 \\
days & $(9.3)$ & $(9.3)$ & $(7.9)$ & $(9.5)$ \\
70 & 57 & 61 & 69 & 36 \\
days & $(11.3)$ & $(13.3)$ & $(8.9)$ & $(10.1)$ \\
\hline
\end{tabular}

age-related sensitivity to acute ethanol using a contextual conditioning procedure. Broadwater and Spear [22] reported that adult rats were more severely disrupted in contextual fear conditioning than were adolescent rats. Results from Experiment 1a, using hippocampus-dependent trace conditioning, might support the opposite prediction that adolescents would be more impaired by pre-training ethanol than adults (see also [16]).

\section{Method}

\subsection{Subjects E Apparatus}

Seventy three Sprague-Dawley rats were used ( $n s=8-10)$. The apparatus as previously described was used.

\subsection{Procedure}

In this experiment, ethanol was administered prior to training via i.p. injection and the vehicle was saline. This change in route of administration was made because of the much shorter duration of training, as well as to be more consistent with previous studies $[11,13,22]$. On the day of training, animals were administered one of 4 doses of ethanol $(0,0.5,1.0$ or $1.5 \mathrm{~g} / \mathrm{kg}$, i.p.). After injection, pairs of animals were kept in holding cages for $10 \mathrm{~min}$. Animals were then placed into the conditioning context and, 2 min later, were given three USs $(0.5 \mathrm{~mA}, 1 \mathrm{~s})$ separated by $30 \mathrm{~s}$. Thirty seconds after the last shock, subjects were removed from the context and returned to the home cage.

Twenty-four hours after training, animals were tested for freezing in the experimental context. Animals were placed in holding cages for $10 \mathrm{~min}$, although no drug was administered prior to test. Animals were then returned to the context and videotaped for $3 \mathrm{~min}$. Videos were scored by a researcher blind to ethanol dose, using a time-sampling procedure. Animals were observed every $10 \mathrm{~s}$ and $\mathrm{a}$ judgment of whether the animal was freezing was made. The data were converted into a percentage of intervals scored as freezing during the test session.

\section{Results and discussion}

In both adolescents and adults, there was no evidence of disrupted context conditioning following pre-training ethanol. The percent freezing scores were analyzed using a 2 (age) $\times 4$ (ethanol dose $) \times 2$ (sex) ANOVA. The analysis yielded no significant effects or interactions [largest $F(1,57)=2.15, p=.148$ ] . Both adolescent and adult subjects exhibited substantial freezing behavior during the context test, and the amount of freezing was unaffected by ethanol at any dose. There was a tendency for adult animals trained with the highest $(1.5 \mathrm{~g} / \mathrm{kg})$ dose to respond with slightly less freezing during the test, but this effect was not statistically significant. The results are shown in Table 1. 


\section{Experiment $\mathbf{2 b}$. contextual fear conditioning in subjects trained with cs-us pairings}

In the previous experiment reported here animals were given USs in the experimental context but no other explicit stimuli were presented; a procedure that could be referred to as foreground contextual conditioning [35]. Many prior studies of ethanol's effects on contextual fear conditioning $[8,11,22]$ instead assessed background context conditioning. That is, animals were given CS-US pairings in the context and conditioning to the context in which CS-US pairings occurred was evaluated. Because this type of training procedure has been found to be sensitive to acute pre-training ethanol in adult rats, a similar procedure was adopted for Experiment $2 \mathrm{~b}$. This permitted us to systematically assess ethanol's impact on foreground as well as background contextual conditioning in the same series of experiments.

\section{Method}

\subsection{Subjects E' apparatus}

The subjects were 40 adolescent (ns $=10$ /group) and 40 adult ( 8 l, ns $=10$ /group) Sprague-Dawley rats. The apparatus was as previously described. Because a tone has commonly been used to assess context conditioning that occurs when a CS and US are paired in the context, we used a 20 -sec tone here similar to that used in prior reports [11,22; see General Method for tone description].

\subsection{Procedure}

Ethanol administration was identical to that described in Experiment 2a. Following a 10-min post-injection period, animals were placed into the experimental context. After a 2 min period of adaptation, subjects received three pairings of a 20 s tone immediately followed by the shock US (.5 mA, $1 \mathrm{~s} ; 60-120 \mathrm{~s} \mathrm{ITI).} \mathrm{Thirty} \mathrm{seconds}$ after the last trial the animals were removed from the context and returned to the home cage. Twenty-four hours later, animals were tested for context freezing as described in Experiment 2a. In addition, all animals were tested for freezing to the tone CS in a novel context approximately $3 \mathrm{~h}$ after the context test. CS freezing was assessed in a manner identical to that described in Experiment 1. Data from 6 adolescent animals was lost due to malfunction of the shock generator. The data from the remaining 34 adolescents and 40 adults were analyzed.

\section{Results and discussion}

Adult rats, particularly females, were impaired in background contextual fear conditioning by acute pre-training ethanol. In contrast, adolescent animals demonstrated no deficit in context conditioning following any dose of ethanol.

\subsection{Context freezing}

The percentage freezing recorded during the 3 min context test was analyzed using a 2 (age) $\times 4$ (dose) $\times 2$ (sex) ANOVA. The analysis yielded a significant main effect of dose $[F(3,58)=5.86, p<.01]$, as well as interactions of Age $\times$ Dose $[F(3,58)=6.05, p<.01]$ and Age $\times$ Dose $\times$ Sex $[F(3,58)=6.58, p<.01]$. Follow-up Dose $\times$ Sex ANOVAs were conducted on the data from each age. For adolescent animals the analysis failed to reveal any significant effects [largest $F(3,26)=1.76, p=.180$ ]. For the adult subjects, the analysis yielded significant main effects of dose $[F(3,32)=15.30, p<.001]$ and sex $[F(1,32)=8.69, p<.01]$, as well as a significant Dose $\times$ Sex interaction $[F(3,32)=6.63, p<.01]$. Subsequent post hoc comparisons a

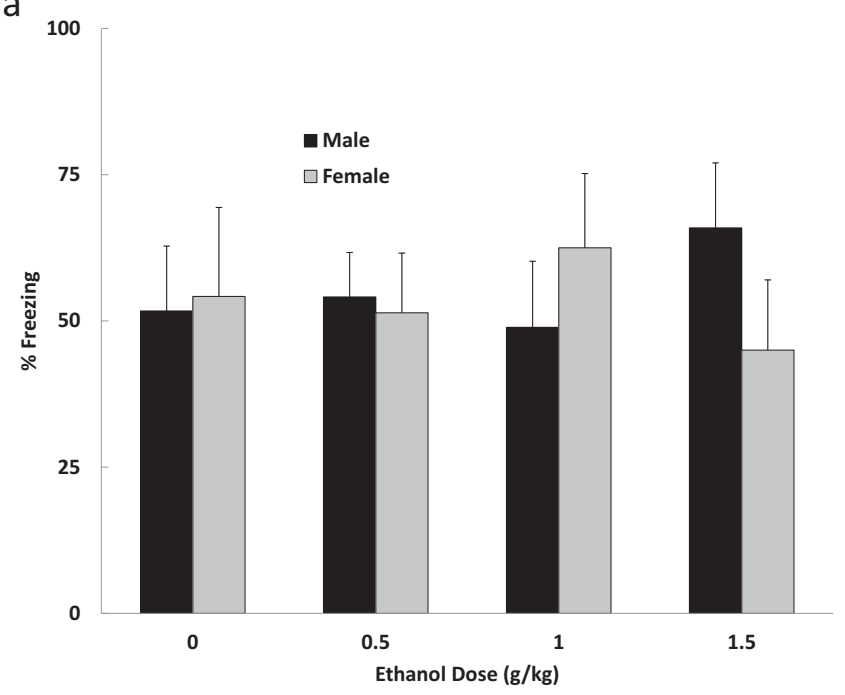

b

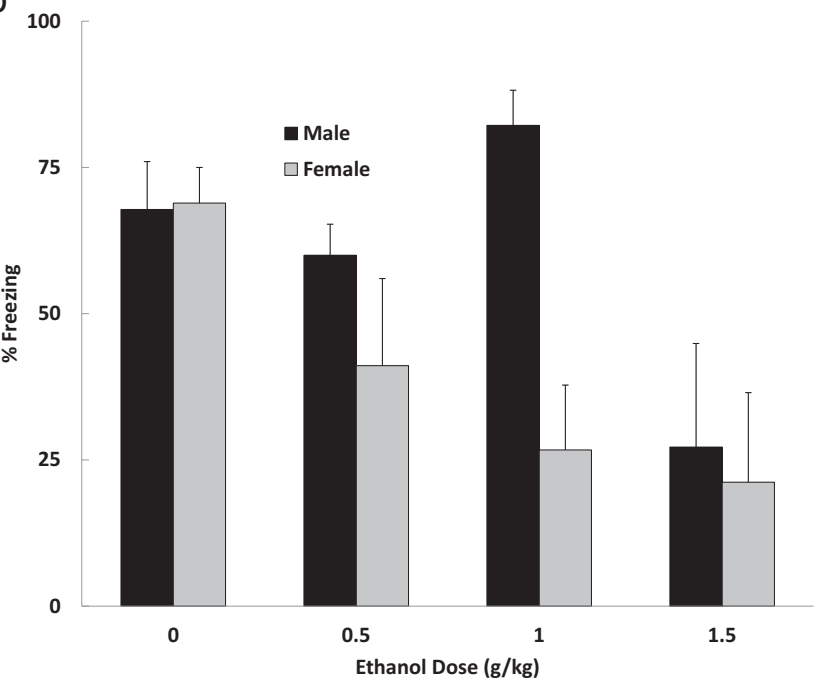

Fig. 3. Mean $( \pm S E M)$ percent freezing during the context test of Experiment $2 b$ Subjects were (a) adolescents or (b) adults that were given $0,0.5,1.0$ or $1.5 \mathrm{~g} / \mathrm{kg}$ ethanol prior to training with tone-shock pairings. Context freezing was assessed $24 \mathrm{~h}$ later. Adolescents were unaffected by pre-training ethanol. In contrast, adults showed an ethanol-related reduction in context fear that was especially pronounced in the females. Adult females exhibited reduced context freezing following pretraining ethanol at $0.5,1.0$ and $1.5 \mathrm{~g} / \mathrm{kg}$, whereas the adult males were affected at the highest dose only.

revealed that adult males exhibited reduced contextual freezing following ethanol administration, but only following the highest dose $(1.5 \mathrm{~g} / \mathrm{kg})$. In contrast, adult females showed disrupted contextual conditioning following all doses of ethanol relative to the $0 \mathrm{~g} / \mathrm{kg}$ controls. These data are shown in Fig. 3a (adolescents) and 3b (adults).

\subsection{CS-elicited freezing}

Responding to the tone CS was analyzed using a 2 (age) $\times 4$ (dose) $\times 2$ (sex) ANOVA. The analysis of pre-CS freezing recorded prior to the first CS presentation resulted in a significant main effect of age $[F(1,54)=6.64, p<.01]$. Adolescents showed more pre-CS freezing $(M=22.4 \pm 4.6 \%)$ than adults $(M=5.88 \pm 4.4 \%)$, as was observed in Experiment 1a.

The analysis of the Change scores recorded during the first test trial revealed a significant main effect of age $[F(1,54)=4.91, p<.01]$, but no effects or interactions involving dose. On average, adult sub- 
jects exhibited higher levels of CS-elicited freezing $(M=51.3 \pm 6.3 \%)$ than adolescents $(M=31.0 \pm 6.5 \%)$, which is likely due to age differences in pre-CS freezing (see above). This pattern was seen across all ethanol doses. Pre-training administration of ethanol had no effect on freezing to the delay-conditioned CS, as was observed in Experiment 1a.

\section{Experiment $2 c$. state-dependent retention in adults}

Experiment 1b suggested that adolescents are sensitive to shifts in internal context that was expressed as a state-dependent deficit in trace conditioning. As part of a more systematic effort to explore conditions under which acute ethanol impairs conditioning by altering memory acquisition versus state-dependent retrieval processes, Experiment 2c examined whether the ethanol-mediated deficit in context conditioning from Experiment $2 \mathrm{~b}$ was also produced by state-dependent shifts from training to test conditions. Because Experiment $2 \mathrm{~b}$ revealed that adult females were more impaired by acute ethanol than males, only female subjects were included in this experiment.

\section{Method}

\subsection{Subjects}

Forty adult female rats (ns $=10$ ) were used and the apparatus was as previously described.

\subsection{Procedure}

The procedure was similar to that of Experiment 2b, except that (1) subjects were injected with 0 or $1.0 \mathrm{~g} / \mathrm{kg}$ ethanol $10 \mathrm{~min}$ prior to training and/or context testing and (2) subjects were not tested for responding to the tone. As in Experiment $1 \mathrm{~b}$ the higher $1.5 \mathrm{~g} / \mathrm{kg}$ dose was not used to prevent hypoactivity that might contaminate scoring of freezing behavior. Groups Saline-Saline and Saline-Ethanol received saline pretreatment prior to the conditioning session and received either saline or ethanol pretreatment, respectively, prior to the test. Groups Ethanol-Saline and Ethanol-Ethanol received ethanol pretreatment prior to conditioning and received either saline or ethanol pretreatment, respectively, prior to test.

\section{Results and discussion}

Adult females trained following acute ethanol displayed lower levels of context conditioning compared with those trained following a saline injection. Moreover, that deficit could not be alleviated when internal state was made similar by additionally administering ethanol prior to the test. Melia et al. [11] reported a similar effect in which acute ethanol disrupted context conditioning, and that disruption could not be accounted for by state-dependent learning.

\subsection{Context freezing}

The percent context freezing data were analyzed using a 2 (training dose) $\times 2$ (testing dose) ANOVA. The analysis yielded a main effect of training dose $[F(1,36)=16.44, p<.01]$. There was no effect or interaction with testing dose. Animals that were trained under the influence of $1.0 \mathrm{~g} / \mathrm{kg}$ ethanol exhibited reduced freezing during the context test $24 \mathrm{~h}$ later compared with saline controls. Ethanol administered prior to test had no effect on levels of freezing. Mean $( \pm$ SEM) percent freezing data are shown in Fig. 4 . These results indicate that ethanol disrupted learning about the context in adult female rats, and that this deficit is not due to the change in drug

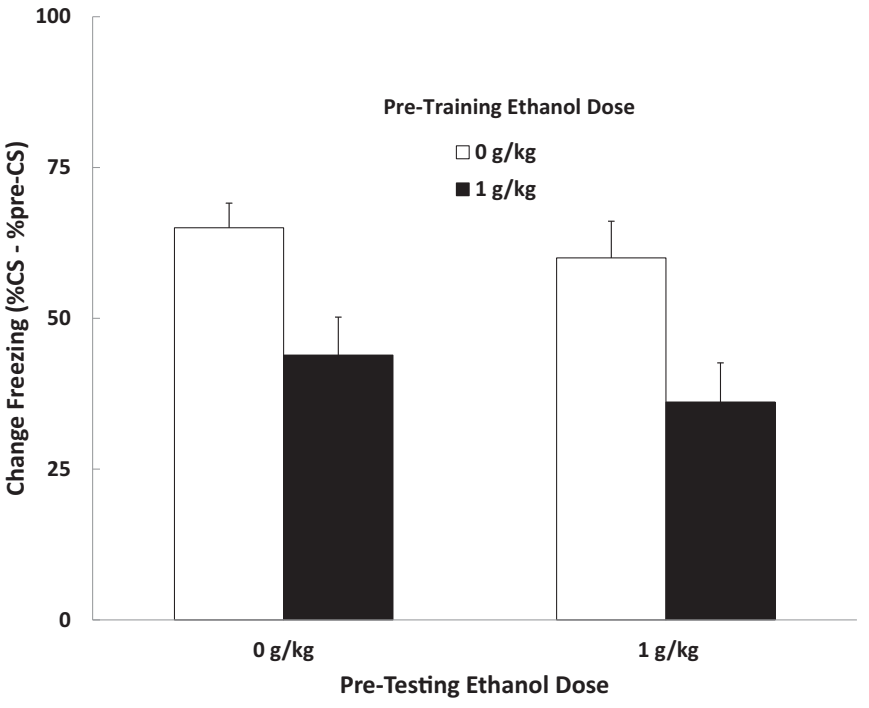

Fig. 4. Results from Experiment 2c showing the effects of pre-training and/or pretesting ethanol administration on contextual fear conditioning in adult female rats. Mean $( \pm$ SEM) percent freezing during the test. Adult female subjects show impaired contextual conditioning when trained under the effects of ethanol. Pre-testing ethanol administration had no effect on context freezing.

state from training to test that might impair responding in a statedependent manner.

\section{General discussion}

These experiments examined age-dependent vulnerabilities to ethanol in hippocampus-dependent memory tasks of trace conditioning and context conditioning. Acute ethanol intoxication disrupted trace conditioning and context conditioning in these experiments and did so in an age-specific manner. Pre-training ethanol disrupted trace conditioning in adolescents more strongly than in adults. When ethanol was administered prior to context conditioning, adults expressed deficits in context conditioning whereas adolescents did not. When an ethanol-mediated deficit was observed, these experiments further explored whether the change in internal state caused by pre-training ethanol compared to the internal state present during test could be responsible for observed conditioning deficits. That is, these experiments uniquely and systematically examined the contribution of state-dependent retrieval deficits to the deleterious effects of ethanol on two hippocampus-dependent memory tasks within the same experimental series. An interesting suggestion from these experiments is that adolescents may be more susceptible to changes in state from training to testing (state-dependent retrieval) than are adults, at least when considering the two different tasks in which the animals exhibited ethanol disruption.

Experiment 1a revealed a unique adolescent sensitivity to effects of pre-training ethanol administration. In that experiment, ethanol produced a deficit in trace conditioning in adolescents at lower doses than was seen in adults. Findings from Experiment $1 \mathrm{~b}$ suggested that the ethanol deficit in trace conditioning in adolescents was mediated by a state-dependent retrieval failure. When pre-training ethanol was accompanied by pre-test ethanol, no deficit in trace conditioning was observed. Thus, pre-training ethanol does not seem to prevent trace conditioning, but rather interferes with the expression of this learning through interactions with internal context and that interference may be enhanced in adolescence. By contrast, adolescents were insensitive to the effects of ethanol in background context conditioning of Experiment $2 \mathrm{~b}$ in which adults were affected. In adults pre-training 
ethanol impaired context conditioning. Experiment 2c revealed that the ethanol-mediated deficit in context conditioning in adult females was not produced by state-dependent changes from training to test. Instead, that deficit is better accounted for as a true deficit in learning or memory encoding. Thus, when deficits in hippocampus-dependent memory were observed, the source of that deficit was state-dependent retrieval failure in adolescents (trace conditioning) but not in adults (background context conditioning) implying enhanced sensitivity of adolescents to changes in internal state.

Other investigators have suggested adolescents are less sensitive than adults to ethanol-produced interoceptive cues. Anderson and Spear [36] exposed rats to positive and negative Pavlovian conditioning discrimination procedures (occasion setting) in which ethanol vs. saline served as a discriminative cue for reward on separate sessions. During acquisition, higher discrimination performance in adults than adolescents was observed which was taken to imply less sensitivity to interoceptive effects of ethanol in adolescents. That finding, however, was present in their positive occasion setting task but not in the negative occasion setting task (p. 189, Fig. 1; see also [37]) and under some conditions (first-trial data only, again for negative occasion setting) adolescents had higher discrimination scores (p. 190, Fig 2b). Other findings from "reactivation" paradigms suggest adolescents may be more sensitive to interoceptive effects of drugs. For example, Roger-Sánchez et al. [38] reported that drug-induced reinstatement after extinction of conditioned place preference produced by cocaine was stronger in adolescent mice than in adults. Thus, variability in memory retrieval may be more sensitive to interoceptive effects of drug exposure in adolescents than adults. Literature directly comparing adolescent and adult ethanol-mediated reinstatement is lacking; however, ethanol-mediated reinstatement in adolescents has been reported [39] and in some cases ethanol-produced reinstatement occurs in rats originally conditioned with ethanol as adolescents but not as adults [40]. Finally, other investigators in both non-human primate [41] and human [42] studies have suggested sensitivity to interoceptive cues (including ethanol [41,p. 341]) declines with age.

Although clear age-dependent effects of acute ethanol were observed in the present studies, because of the change in route of administration (i.g. in Experiment 1 Trace Conditioning; i.p. in Experiment 2 Context Conditioning) direct comparison across studies should be made with caution. Blood ethanol concentration (BEC) is typically lower following intragastric compared to intraperitoneal administration [43]. It is possible that lower BEC promotes state-dependency in adolescents. Thus in Experiment 1 lower BEC promoted state-dependency in adolescence with Trace conditioning (i.g.) but not in Experiment 2 with Context conditioning (i.p.) where BEC was presumably higher. Yet, the deficit in Trace conditioning (see Fig. 1a) revealed to be state-dependent in Experiment $1 \mathrm{~b}$ increased (not decreased) as dose increased. That is, if lower BEC were associated with stronger state-dependency in adolescents a pattern opposite to that observed might have been predicted. The present studies did not record BEC. Therefore this remains a viable alternative. Additionally, weaker discriminative stimulus control by ethanol despite higher BEC in older compared to younger primates has also been reported [41][cf. 41] suggesting that variation in BEC does not uniquely predict the conditions under which ethanol's interoceptive cues will control behavior.

It seems contradictory that ethanol impaired hippocampusdependent trace conditioning in adolescents more strongly than in adults and yet ethanol impaired hippocampus-dependent context conditioning in adults but not in adolescents in the present experiments. If the hippocampus is required for both of these types of fear learning [27,35], and adolescents express enhanced sensitivity to disrupting effects of ethanol in one task, it follows that adolescents should similarly express enhanced sensitivity to the disrupting effects of ethanol in other hippocampus-dependent tasks. This was not the case here. Interestingly, this pattern models similar contradictions noted in the literature. Broadwater and Spear [22] reported that adult rats were more impaired by acute ethanol in context conditioning compared to adolescents, but other studies using the Morris water maze spatial task observed greater disruption by ethanol in adolescents compared to adults [16] These "contradictions" are important because they reveal that all hippocampus-dependent tasks are not equivalent in susceptibility to ethanol amnesia and therefore that the regional nature of hippocampus involvement in these tasks is not uniform. Moreover, the present research implies that sensitivity of regionally specific pathways within the hippocampus to ethanol is not uniform in development.

Several studies have suggested the importance of the dorsal hippocampus to context conditioning [35,44,45]. Some evidence suggests a critical role of ventral but not dorsal hippocampus in trace conditioning. Yoon and Otto [46] for example, demonstrated pre-training lesions of the ventral hippocampus disrupted trace conditioning but lesions to the dorsal hippocampus did not. Czerniawski, Yoon, \& Otto [47] found that temporary inactivation of ventral but not dorsal hippocampus impaired acquisition of trace conditioning but that dorsal and not ventral inactivation impaired performance on a spatial task. Thus, one possibility is that dorsal and ventral hippocampus involvement vary depending on task demands in context and trace conditioning. Findings from the present experiment would suggest further that vulnerability of these hippocampus regions to the disruptive effects of ethanol is not the same in adolescence and adulthood. Namely, based on the present findings perhaps ventral hippocampus (trace conditioning; but see $[28,48]$ ) is more susceptible to ethanol disruption in adolescence but that dorsal hippocampus (context conditioning) is more susceptible to ethanol disruption in adulthood. This organizing scheme warrants further study and remains speculative. Moreover, other evidence concerning hippocampus involvement in state-dependent learning poses challenges to such a hypothesis. Rezayof et al. [49] reported evidence suggesting that ethanol state-dependent learning may rely, in part, on the dorsal hippocampus. In that research, which employed a passive avoidance task, administration of NMDA to the dorsal hippocampus facilitated ethanol state-dependent retrieval while the NMDA antagonist MK801 impaired ethanol state-dependent retrieval. Given evidence that NMDA receptor expression is developmentally regulated [50], heightened sensitivity to state-dependent learning effects might occur during different developmental periods. Though to our knowledge no studies have directly compared the relative contribution of dorsal versus ventral involvement in ethanol statedependent memory, the implication is nonetheless that the dorsal hippocampus is involved in modulating state-dependent learning (see also [51,52]). In the studies reported here, we observed strong state-dependency in the trace conditioning task which has been suggested to be ventrally mediated $[46,47]$ and not in context conditioning which has been suggested to be dorsally mediated [45]. Thus an appeal to ethanol disrupting ventral hippocampus more in adolescence, perhaps accounting for the presently observed adolescent ethanol deficit in trace conditioning, would seem to be at odds with the suggested role of the dorsal hippocampus in context conditioning and state-dependency where no adolescent ethanolproduced deficit, and no state-dependency, was observed. Despite significant advances in understanding the role of the hippocampus in memory $[44,53,54]$ these conflicts remain more interesting than resolved. But what they bring focus to is the strong need for studies which, in the same series of experiments, permit direct comparison of different forms of hippocampus-dependent memory [4]. Given convergent evidence of age-specific alteration in hippocampus sensitivity to ethanol, continued analysis of how 
developmental trajectory alters sensitivity to ethanol may serve as a powerful gateway to better isolating the role of the hippocampus in learning.

\section{References}

[1] R.S. Ryback, The continuum and specificity of the effects of alcohol on memory, Q. J. Stud. Alcohol 32 (1971) 995-1016.

[2] R.G. Lister, C. Gorenstein, D. Risher-Flowers, Dissociation of the acute effects of alcohol on implicit and explicit memory processes, Neuropsychologia 29 (1991) 1205-1212.

[3] T. Duka, R. Weissenborn, Z. Dienes, State-dependent effects of alcohol on recollective experience, familiarity and awareness of memories, Psychopharmacol. (Berl) 153 (2001) 295-306.

[4] D.B. Matthews, J.R. Silvers, The use of acute ethanol administration as a tool to investigate multiple memory systems, Neurobiol. Learn. Mem. 82 (2004) 299-308.

[5] A.M. White, D.B. Matthews, P.J. Best, Ethanol, memory, and hippocampal function: a review of recent findings, Hippocampus 10 (2000) 88-93.

[6] D.B. Matthews, A.L. Morrow, S. Tokunaga, J.R. McDaniel, Acute ethanol administration and acute allopregnanolone administration impair spatia memory in the Morris water task, Alcoholism: Clin. Exp. Res. 26 (2002) 1747-1751

[7] A.M. White, T.M. Elek, T.L. Beltz, P.J. Best, Spatial performance is more sensitive to ethanol than nonspatial performance regardless of cue proximity, Alcoholism: Clin. Exp. Res. 22 (1998) 2102-2107.

[8] A.Z. Weitemier, A.E. Ryabinin, Alcohol-induced memory impairment in trace fear conditioning: a hippocampus-specific effect, Hippocampus 13 (2003) 305-315.

[9] T.J. Gould, Ethanol disrupts fear conditioning in C57BL/6 mice, J. Psychopharmacol. 17 (2003) 77-81.

[10] D.J. McKinzie, J. Lee, J.H. Bronfen, L.P. Spear, N.E. Spear, Context and tone conditioning are selectively impaired by ethanol in the preweanling rat: effects of dose and time of administration, Behav. Neural Biol. 62 (1994) 201-209.

[11] K.R. Melia, A.E. Ryabinin, K.P. Corodimas, M.C. Wilson, J.E. LeDoux, Hippocampal-dependent learning experience-dependent activation of the hippocampus are preferentially disrupted by ethanol, Neuroscience 74 (1996) 313-322.

[12] R.B. Berry, D.B. Matthews, Acute ethanol administration selectively impairs spatial memory in C57BL/6J mice, Alcohol 32 (2004) 9-18.

[13] C. Land, N.E. Spear, Fear conditioning is impaired in adult rats by ethano doses that do not affect periadolescents, Int. J. Dev. Neurosci. 22 (2004) 355-362.

[14] V.S. Chin, C.E. Van Skike, D.B. Matthews, Effects of ethanol on hippocampa function during adolescence: a look at the past and thoughts on the future, Alcohol 44 (2010) 3-14.

[15] L.P. Spear, Adolescents and alcohol: acute sensitivities, enhanced intake, and later consequences, Neurotoxicol. Teratol. 41 (2014) 51-59.

[16] B.J. Markwiese, S.K. Acheson, E.D. Levin, W.A. Wilson, H.S. Swartzwelder, Differential effects of ethanol ion memory ion adolescent and adult rats, Alcoholism: Clin. Exp. Res. 22 (1998) 416-421.

[17] V.S. Chin, C.E. Van Skike, R.B. Berry, R.E. Kirk, J. Diaz-Granados, D.B. Matthews, Effects of acute ethanol and acute allopregnanolone on spatial memory in adolescent and adult rats, Alcohol 45 (2011) 473-483.

[18] C. Land, N.E. Spear, Ethanol impairs memory of a simple discrimination in adolescent rats at doses that leave adult memory unaffected, Neurobiol. Learn. Mem. 81 (2004) 75-81.

[19] G.K. Pyapali, D.A. Turner, W.A. Wilson, H.S. Swartzwelder, Age and dose-dependent effects of ethanol on the induction of hippocampal long-term potentiation, Alcohol 19 (1999) 107-111.

[20] H.S. Swartzwelder, W.A. Wilson, M.I. Tayyeb, Differential sensitivity of NMDA receptor-mediated synaptic potentials to ethanol in immature versus mature hippocampus, Alcoholism: Clin. Exp. Res. 19 (1995) 320-323.

[21] H.S. Swartzwelder, W.A. Wilson, M.I. Yayyeb, Age-dependent inhibition of long-term potentiation by ethanol in immature versus mature hippocampus, Alcoholism: Clin. Exp. Res. 19 (1995) 1480-1485.

[22] M. Broadwater, L.P. Spear, Age differences in fear retention and extinction in male Sprague-Dawley rats: effects of ethanol challenge during conditioning, Behav. Brain Res. 252 (2013) 377-387.

[23] D.A. Overton, Drug state dependent learning, in: M.E. Jarvik (Ed.) Psychopharmacology in the Practice of Medicine, Appleton-Century-Crofts, New York, 1977, pp. 73-79.

[24] J.E. LeDoux, Emotion circuits in the brain, Ann. Rev. Neurosci. 23 (2000) $155-184$

[25] D. Ivkovich, M.E. Stanton, Effects of early hippocampal lesions on trace, delay, and long-delay eyeblink conditioning in developing rats, Neurobiol. Learn. Mem. 76 (2001) 426-446.
[26] T. Kaneko, R.F. Thompson, Disruption of trace conditioning of the nictitating membrane response in rabbits by central cholinergic blockade, Psychopharmacol. (Berl) 131 (1997) 161-166.

[27] M.D. McEchron, H. Bouwmeester, W. Tseng, C. Weiss, J.F. Disterhoft, Hippocampectomy disrupts auditory trace fear conditioning and contextual fear conditioning in the rat, Hippocampus 8 (1998) 638-646.

[28] J.J. Quinn, S.S. Oommen, G.E. Morrison, M.S. Fanselow, Post-training excitotoxic lesions of the dorsal hippocampus attenuate forward trace, backward trace, and delay fear conditioning in a temporally specific manner, Hippocampus 12 (2002) 495-504.

[29] P.S. Hunt, R. Richardson, Pharmacological dissociation of trace and long delay fear conditioning in young rats, Neurobiol. Learn. Mem. 87 (2007) 86-92.

[30] R.E. Clark, J.R. Manns, L.R. Squire, Trace and delay eyeblink conditioniong: contrasting phenomena of declarative and nondeclarative memory, Psychol. Sci. 12 (2001) 304-308

[31] R.E. Clark, J.R. Manns, L.R. Squire, Classical conditioning, awareness, and brain systems, Trends Cognit. Neurosci. 6 (2002) 524-531.

[32] R.C. Barnet, P.S. Hunt, Trace and long-delay fear conditioning in the developing rat, Learn. Behav. 33 (2005) 437-443.

[33] M.S. Fanselow, Conditional and unconditional components of post-shock freezing, Pavlov. J. Biol. Sci. 15 (1980) 177-182.

[34] S.G. Anagnostaras, S. Maren, M.S. Fanselow, Scopolamine selectively disrupts the acquisition of contextual fear conditioning in rats, Neurobiol. Learn. Mem. 64 (1995) 191-194.

[35] R.G. Phillips, J.E. LeDoux, Differential contribution of amygdala and hippocampus to cued and contextual fear conditioning, Behav. Neurosci. 106 (1992) 274-285.

[36] R.I. Anderson, L.P. Spear, Age differences in ethanol discrimination: acquisition and ethanol dose generalization curves following multiple training conditions in adolescent and adult rats, Alcoholism: Clin. Exp. Res. 38 (2014) 186-194.

[37] C.H. Groseclose, L.D. Middaugh, The discrimination and durability of an ethanol cue in young and mid-aged female mice, Alcohol 14 (1997) 191-197.

[38] M.A. Roger-Sánchez Aguilar, M. Rodríguez-Arias, C.M. Aragon, J. Miñarro, Age and sex-related differences in the acquisition and reinstatement of CPP in mice, Neurotoxicol. Teratol. 34 (2012) 101-115.

[39] H.C. Brenhouse, S.L. Anderson, Delayed extinction and stronger reinstatement of cocaine conditioned place preference in adolescent rats, compared to adults, Behav. Neurosci. 122 (2008) 460-465.

[40] M.R.R. Pascual Do Couto, S. Alfonso-Loeches, M.A. Aguilar, M. Rodrigues-Arias, Change in histone acetylation in the prefrontal cortex of ethanol-exposed adolescent rats are associated with ethanol-induced place conditioning, Neuropharmacology 62 (2012) 2309-2319.

[41] C.M. Helms, K.A. Grant, The effect of age on the discriminative stimulus effects of ethanol and its GABAA receptor mediation in Cynomolgus monkeys, Psychopharmacology (Berl) 216 (2011) 333-343.

[42] S. Khalsa, D. Rudrau, D. Tranel, Interoceptive awareness declines with age, Psychophysiology 46 (2009) 1130-1136.

[43] D.J. Livy, S.E. Parnell, J.R. West, Blood ethanol concentration profiles: a comparison between rats and mice, Alcohol 29 (2003) 165-171.

[44] R.C. O'Reilly, J.W. Rudy, Conjunctive representations in learning and memory: principles of cortical and hippocampal function, Psychol. Rev. 108 (2001) $311-345$.

[45] P. Matus-Amat, E.A. Higgins, R.M. Barrientos, J.W. Rudy, The role of the dorsal hippocampus in the acquisition and retrieval of contextual memory representations, J. Neurosci. 24 (2004) 2431-2439.

[46] T. Yoon, T. Otto, Differential contributions of dorsal vs. ventral hippocampus to auditory trace fear conditioning, Neurobiol. Learn. Mem. 87 (2007) 464-475.

[47] J. Czerniawski, T. Yoon, T. Otto, Dissociating space and trace in dorsal and ventral hippocampus, Hippocampus 19 (2009) 20-32.

[48] N. Chowdhury, J.J. Quinn, M.S. Fanselow, Dorsal hippocampus involvement in trace fear conditioning with long, but not short, trace intervals in mice, Behav. Neurosci. 119 (2005) 1396-1402.

[49] A. Rezayof, K. Sharifi, M.R. Zarrindast, Y. Rassouli, Modulation of ethanol state-dependent learning by dorsal hippocampal NMDA receptors in mice, Alcohol 42 (2008) 667-674.

[50] M.V. Johnston, Neurotransmitters and vulnerability of the developing brain, Brain Dev. 17 (1995) 301-306.

[51] A. Rezayof, M. Tahmineh, Y. Rassouli, M.R. Zarrindast, Dorsal hippocampa dopamine receptors are involved in mediating ethanol state-dependent memory, Life Sci. 80 (2007) 285-292.

[52] A. Rezayof, S. Alijanpour, M.R. Zarrindast, Y. Rassouli, Ethanol state-dependent memory: involvement of dorsal hippocampal muscarinic and nicotinic receptors, Neurobiol. Learn. Mem. 89 (2008) 441-447.

[53] H. Eichenbaum, Memory systems, in: R.J. Nelson, S.J.Y. Mizumori, I.B. Weine (Eds.), Handbook of Psychology: Behavioral Neuroscience, Vol. 3, John Wiley \& Sons, NY, 2013, pp. 551-573.

[54] J. O'Keefe, L. Nadel, Hippocampus as a Cognitive Map, Oxford University Press., 1978. 